\title{
The Expanding Universe: Dark Energy
}

Don Lincoln and Brian Nord, Fermi National Accelerator Laboratory, Batavia, IL 60510

As is true of a far more famous story, it all began a long time ago, in a galaxy far, far away. It even involved a binary star system. A small star, called a white dwarf, had become a burned out husk of its former self and it turned to gorging on hydrogen and helium from its bloated red giant neighbor. The transferred gas reignited the fires of the white dwarf until the temperature from the fusion reaction proved too much for the gravity that struggled to contain it. In the blink of an eye, the star detonated in a supernova, a cosmic maelstrom seen perhaps only once per century in a typical galaxy.

The death cry of the star roared across the heavens in a blinding sphere of light that spread throughout the cosmos, dimming as it expanded. Photons traversed the universe for eons until about two decades ago, when they entered the apertures of mankind's telescopes and told their tales - a bittersweet story of stellar death and the much grander saga of the life and history of the universe itself. The photons brought with them news that the make-up of the cosmos is different than scientists expected that perhaps there is an energy field permeating all of space and time. This recent discovery greatly alters our understanding of the composition, the origin, and the very destiny of the universe. This energy field is often called dark energy and understanding it is one of the most pressing questions in modern cosmology. 


\section{History: An Expanding Story}

In 1916, Einstein published his theory of General Relativity (GR), a dramatic and revolutionary new formulation of gravity. His theory described a dynamic universe with spacetime distorting due to the presence of energy and matter in the universe: space tells matter how to move, and matter and energy tell spacetime how to curve. This theory is an improvement on Newtonian gravity, which failed to accurately predict the orbit of Mercury and the degree of light deflection during a solar eclipse.

When applied to the universe as a whole - first by George Lemaitre, Alexandre Friedmann and others - GR implied that the universe started from a single, small cataclysmic event; what's more, it predicted that if there was a sufficient amount of gravitating mass, that mass could pull the universe back in on itself. This prediction of a dynamic cosmos ran afoul of the theoretical consensus of the time, which held that the cosmos was eternal and unchanging, i.e. the steady state theory. To comply with this theoretical prejudice of a static cosmos, and in order to counteract the attractive force of gravity, Einstein added to his equations a term called the cosmological constant, $\Lambda$. This term was intended to stabilize the universe against contraction.

By studying nearby galaxies, Edwin Hubble showed in 1929 that typically galaxies are receding from Earth. What's more, the farther away a galaxy lies from us, the faster it is moving. The physical mechanism underlying this relationship involves the expansion of space. This is a subtle and crucial point. The galaxies themselves are not moving through space; rather space itself is expanding, carrying away the galaxies with it. This statement is substantially true, but not completely. The velocities of galaxies in the vicinity of the Milky Way are in random directions and are on the order of one or two 
hundred miles per second. The expansion characterized by Hubble is about 41 miles per second per megaparsec distance from Earth. Thus by about five megaparsecs of distance, the expansion rate is about 200 miles per second, or enough to overcome the random local velocities of galaxies. At distances beyond about ten megaparsecs or so, the Hubble expansion is dominant. The observation of an expanding universe effectively killed the idea of a static universe and raised the credibility of the Big Bang as the prevailing theory of cosmic origins. Einstein dropped his cosmological constant and called it his "biggest blunder."

In the Big Bang theory, the cosmos began 13.8 billion years ago in a much smaller and hotter state than it is in today. It has been expanding and cooling ever since. As soon as the theory was proposed, a vigorous and multi-decade debate began regarding the expansion history and future of the cosmos. Hubble's original work only covered distances to about six million light-years from Earth and revealed that locally, the relationship is simple and linear. Hubble's discovery can be summarized by the relationship, $v=H d$, where $v$ is the velocity of the galaxy, $d$ is the distance, and $H$ is Hubble's constant of proportionality. In the intervening century, scientists have pushed the distance back to billions of light-years, showing that when more distant galaxies are taken into account, the data reveal a more complicated expansion history, and that $\mathrm{H}$ has a strong dependence on the distance from Earth and, consequently, on time.

The next question became, what is the fate of the universe? How will it evolve in the future? It was thought there were three possibilities. If there is enough matter in the universe, then the matter's self-gravity would cause space on large scales to close in on itself, resulting in an eventual 'big crunch' (closed universe). If there's not enough 
matter, space could expand forever, slowing but never stopping (an open universe). In a Goldilocks scenario, there could be just the right amount of matter-energy, in which space would expand forever with an expansion rate asymptotically approaching zero (a flat universe).

The names given to these different scenarios highlight the intimate connection between the overall geometry of the universe, its matter-energy content, and its ultimate fate. A flat universe follows Euclidean mathematics and the sum the angles of a triangle add to $180^{\circ}$. As illustrated in figure 1, in a closed (spherical) universe, the sum of the angles add to a number greater than $180^{\circ}$; in contrast, for an open (hyperbolic) universe, the sum adds to a number less than $180^{\circ}$. This connection means that determining the shape of the cosmos will simultaneously establish the integrated amount of mass and energy in the universe.

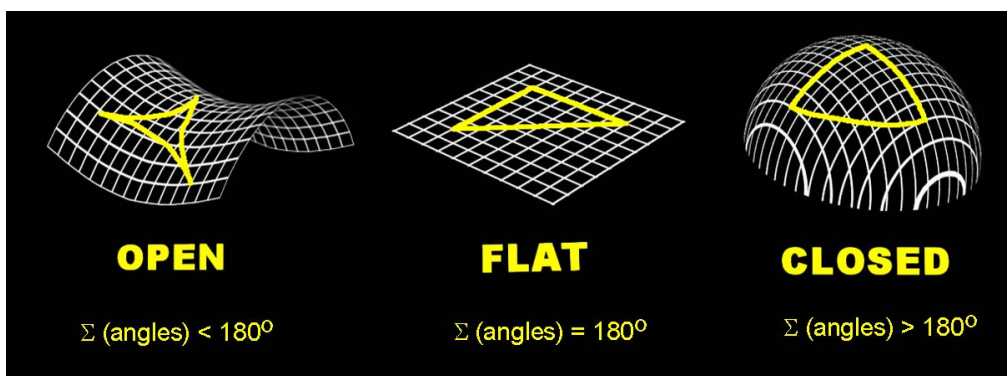

Figure 1: The shape of space determines the sum of the angles of a triangle. In the familiar Euclidean space, this sum is $180^{\circ}$; however this is not true for curved spaces. In an open (hyperbolic) space, the sum is lower than the Euclidean space, while for a closed geometry, the sum is larger.

[File: angles.jpg]

Determining the shape of the universe can be accomplished using a clever technique. Since 1964, astronomers have known of the Cosmic Microwave Background 
(CMB)[Reference: $\mathrm{CMB}$, which is a direct image of the conditions of the cosmos approximately 380,000 years after the Big Bang, when the universe cooled enough to become transparent to light and thus the conditions of the universe at that time. While the CMB is extremely uniform, in 1992 the COBE[Reference: COBE] collaboration announced that they had detected a residual non-uniformity of about one part in 100,000. Subsequent measurements by the WMAP and Planck[Reference: Planck] satellites confirmed and extended this observation. The size of these non-uniformities reflected conditions of the early universe. Combining the observed angular separation of the non-uniformities, the distance of the source of the CMB radiation, and general relativity, cosmologists were able to establish that the geometry of the universe is consistent with being flat[Reference:Flat]. The approach is illustrated in figure 2 . Given that a flat universe corresponds to a unique quantity of matter and energy, this measurement determined the energy content of the universe. However, this determination of the energy content (and, by extension, mass content and expansion history) of the universe was only inferential and a direct measurement was preferable. 


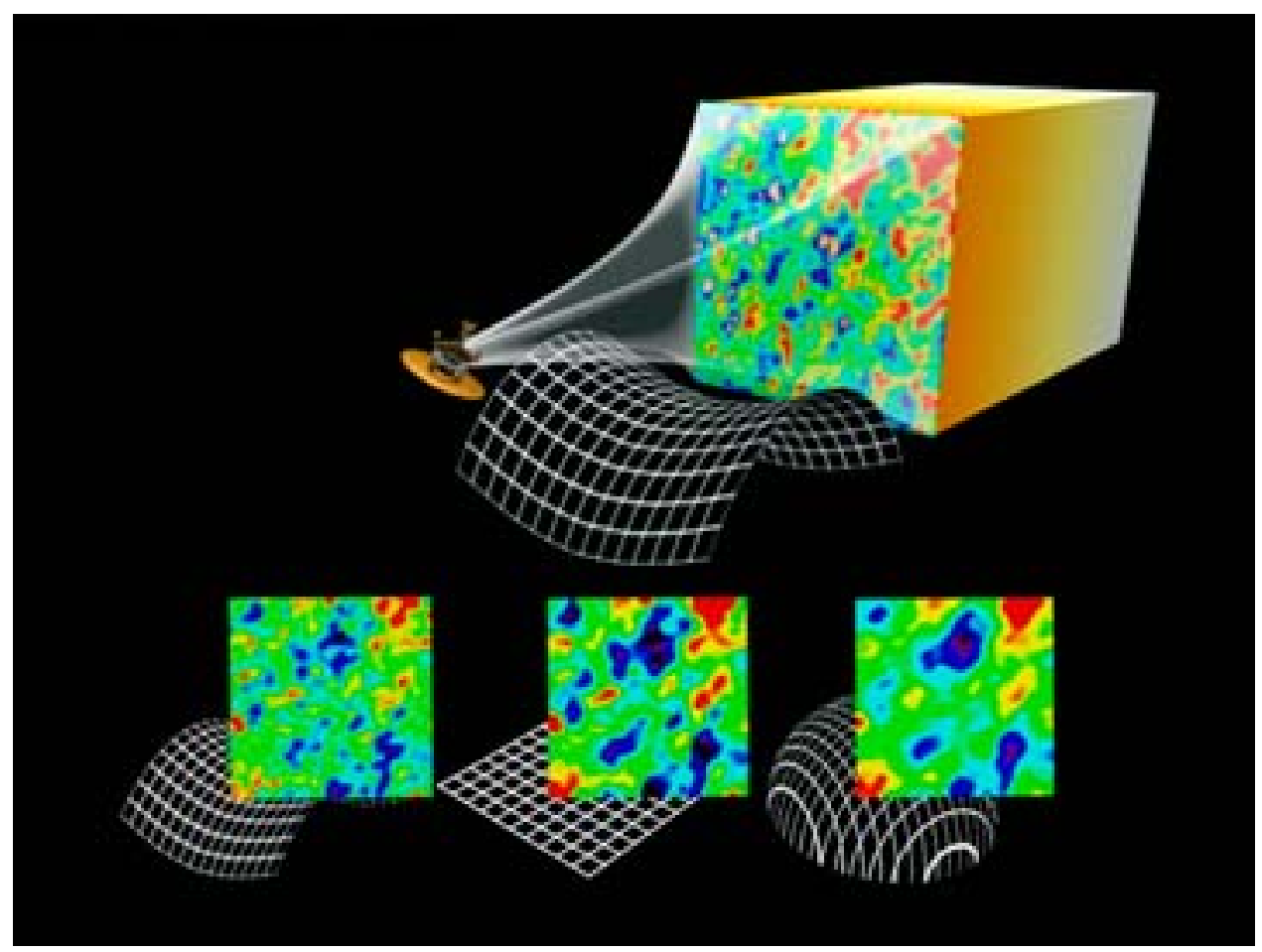

Figure 2: By studying the temperature variations in the cosmic microwave background, scientists are able to determine the geometry of space itself. If the universe were flat the separation between large adjacent spots would be about 1 degree, while for a closed (open) universe, the separation would be larger (smaller). Experimentally, the spots appear to be separated by about a degree to within an uncertainty less than one percent. [Figure credit: Credit: NASA / WMAP Science Team]

[URL: http://wmap.gsfc.nasa.gov/media/030639/030639_1_720.png

[File: geometry.jpg]

Large galaxy surveys, like the CfA redshift survey [Reference:CFA] and the Sloan Digital Sky Survey [Reference:SDSS] performed from the late 1970s through the early 2000's, were able to measure the mass of the universe and found it to be far too low to be consistent with a flat cosmos. Even including dark matter [Reference:Dark Matter], the amount of mass in the universe seems to be about only a third of that needed. Given the compelling measurements of the geometry of space, it was clear that there was a mystery and the universe contained mass and energy that had not 
been identified. Resolving this conundrum would require a precise understanding of the expansion history of the universe.

\section{Experimental technique}

In order to directly determine the expansion history and future of the universe, astronomers needed to study objects located at a range of distances from Earth and determine both their distance and their velocity. This is a very difficult task and many considerations need to be taken into account to be successful.

The first thing that was needed was to find a population of very remote objects. We would then compare the objects' known brightnesses (or intensities, I) to their observed brightnesses $\left(I_{0}\right)$ and infer the distances. While the requirements of general relativity dictate a more complex algorithm is employed, this is conceptually similar to employing the familiar $\mathrm{I}=\mathrm{I}_{0} /\left(4 \pi \mathrm{r}^{2}\right)$. Galaxies are visible distant objects, but the problem is that an individual galaxy's intrinsic brightness is unknown and therefore they are unsuitable for this measurement. However, there exists an astronomical event for which the intrinsic brightness can be determined - exploding stars of the type that opened this article. Much like the galaxies that Hubble observed, supernovae serve as buoys in the fabric of spacetime to reveal the speed of spatial expansion (and any changes in it). These Type la Supernovae are formed in binary star systems in which one is a white dwarf. As shown in figure 3 , the white dwarf siphons off matter from its companion until the white dwarf's mass reaches about 1.38 times the mass of our sun. At that mass, the star explodes in a supernova. This process results in a very bright and predictable initial light source, corresponding to an object that, were it to explode at 
a distance of 70 light years from earth, would appear to be about 100 times brighter than a full moon. For cosmological distance measurements, Type la supernovae are the preferred objects of study.

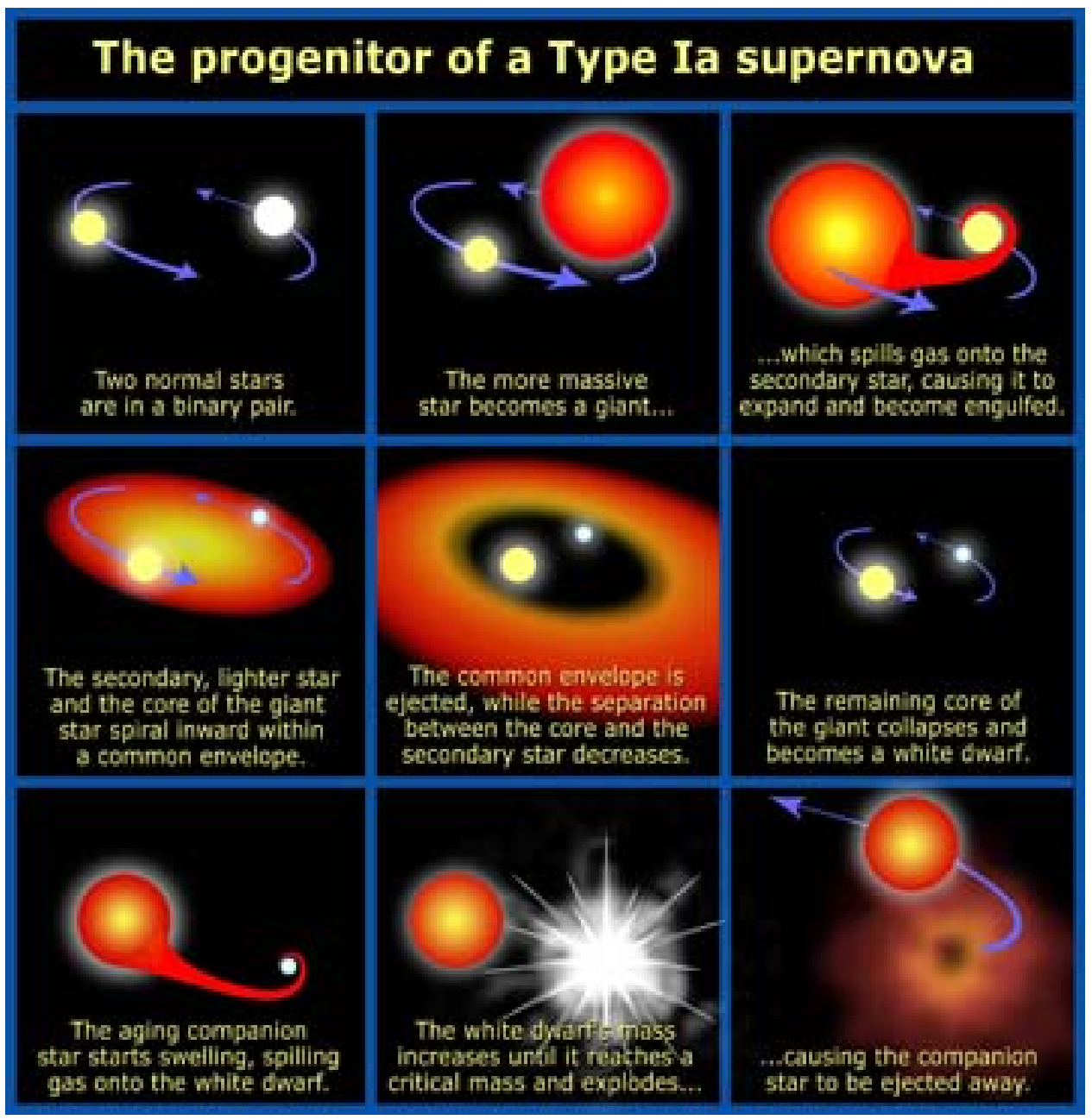

Figure 3: A Type 1a Supernova begins with a binary star system. The white dwarf siphons the gas from the other star, and eventually becomes too dense to remain stable and explodes, ejecting its contents and what remains of its companion into the interstellar medium. (Illustration credit: NASA, ESA, and A. Field (STScI))

[URL: http://www.pha.jhu.edu/ bfalck/laprogenitor.jpg]

[File: supernova_process.jpg] 


\section{The hunt}

Comparing an object's distance to its recessional velocity is a crucial step. By repeating this procedure over a range of distances, using both nearby and far-away supernovae, scientists hoped to understand in detail the universe's expansion history. In the 1990s, two competing teams investigated this topic. They were the Supernova Cosmology Project[Reference:SCP], headed by Saul Perlmutter and the High-Z Supernova Search Team [Reference:High-Z]headed by Brian Schmidt and Adam Riess. By studying fewer than a hundred supernovae, the two teams were able to map out the past velocity history of the cosmos. In contrast with the simplest expectations, they found out that not only was the universe expanding, but the expansion was accelerating. Figure 4, which compares the brightness of supernovae to the size of the universe, illustrates the key features of their observations. The observations were published in 1998 and led to Perlmutter, Riess and Schmidt sharing the 2011 Nobel Prize in physics.

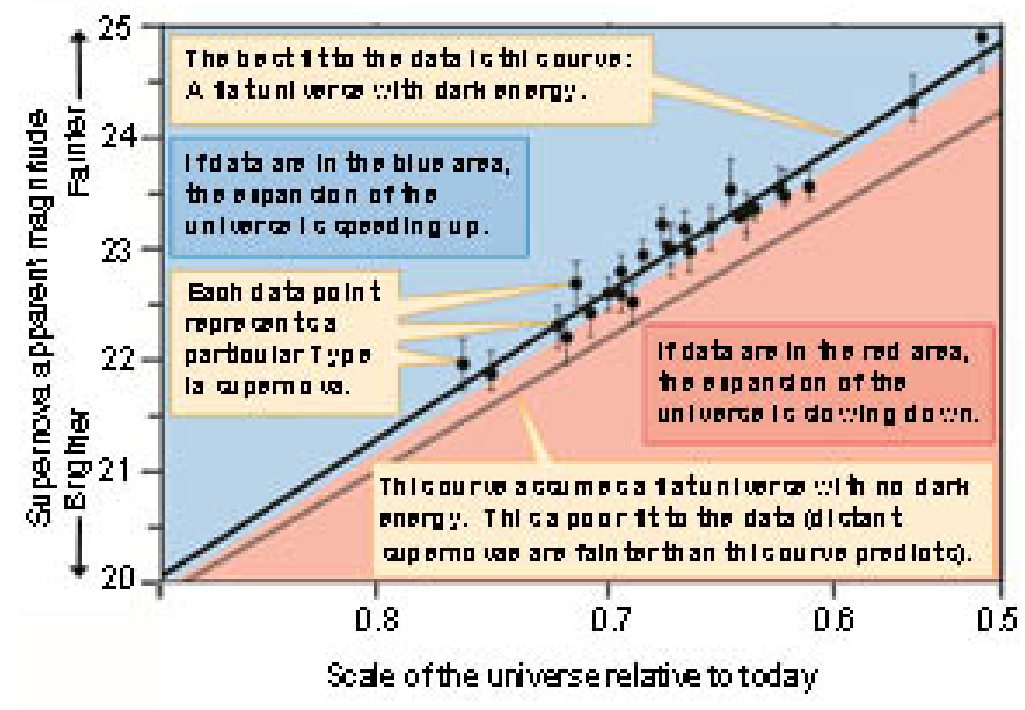


Figure 4: The brightnesses of a sample of supernova as a function of the scale of the universe (or, equivalently, time in the past), overlaid on theoretical predictions from various possible cosmologies. Samples like this were used to discern that we live in a universe in which the expansion is speeding up. [Adapted from figure by Dmitri Pogosyan.]

[File: size_vs_brightness.jpg]

\section{Paradigm shift}

The realization that the expansion of the universe is accelerating required an explanation. The observed flatness of space and measured mass content of the universe suggested that there was a missing mass or energy component of the universe, but the accelerating expansion required this energy be of a different form. In fact, it seemed that Einstein's cosmological constant, which could overcome the attractive properties of familiar gravity, might just fit the bill. Thus the cosmological constant has experienced a renaissance. Imagine if Einstein hadn't removed this term from his equations. The guy could have been famous.

Physicist Michael Turner coined the phrase "dark energy" to describe the simplest possible addition to general relativity that could explain observations. In the model, dark energy is a property of space itself and has an energy density that is constant throughout both space and time. This density is very low. Using the massenergy equivalence formula, the current estimate for the value of dark energy is $1.67 \times$ $10^{-27} \mathrm{~kg} / \mathrm{m}^{3}$, or about a proton per cubic meter. This modest amount of energy contributes only a very small amount to the energy budget of the universe in small 
volumes (it amounts to about six tons in a sphere with a circumference equal to the orbit of Pluto), but when projected to the universe as a whole, dark energy appears currently to be about $69.7 \%$ of the energy budget of the universe. (The remaining components come from ordinary matter composed of atoms, $4.7 \%$, and dark matter, $25.7 \%$.) The constant density of dark energy has profound consequences for its role in the cosmos.

The most familiar density is that of matter: if both the amount of matter and its enclosing volume remain fixed, so will the density. However, if the volume increases, as it does in an expanding universe, while the matter stays unchanged, then the matter density will decrease proportional to the amount that the volume of space has increased. We directly observe this phenomenon over cosmic history. This is true for both dark and ordinary matter.

On the other hand, dark energy is presumed to be a property of space itself and its density is constant. Therefore, if the amount of space increases, the amount of dark energy increases. In an expanding universe, the constant dark energy density will eventually exceed the matter density, which is ever-decreasing. In our universe, this transition happened 8.5 billion years ago. In the very early universe, dark energy played no significant role at all, as quantified in figure 5. Figure 6 illustrates the growth history of the universe over cosmic timescales. The flaring of the size of the universe beginning at the center of the figure and moving towards the right shows the effect of dark energy. 


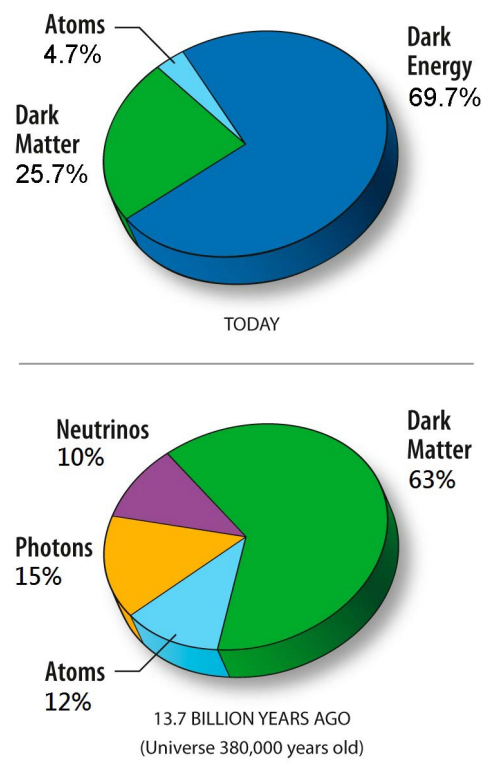

Figure 5: The mix of components of the universe (dark energy, dark matter, atoms, photons and neutrons) has evolved over the universe's history. [Figure credit: Adapted from the Wikipedia.]

[File: Pie_chart_DM-DE-OM.jpg] 


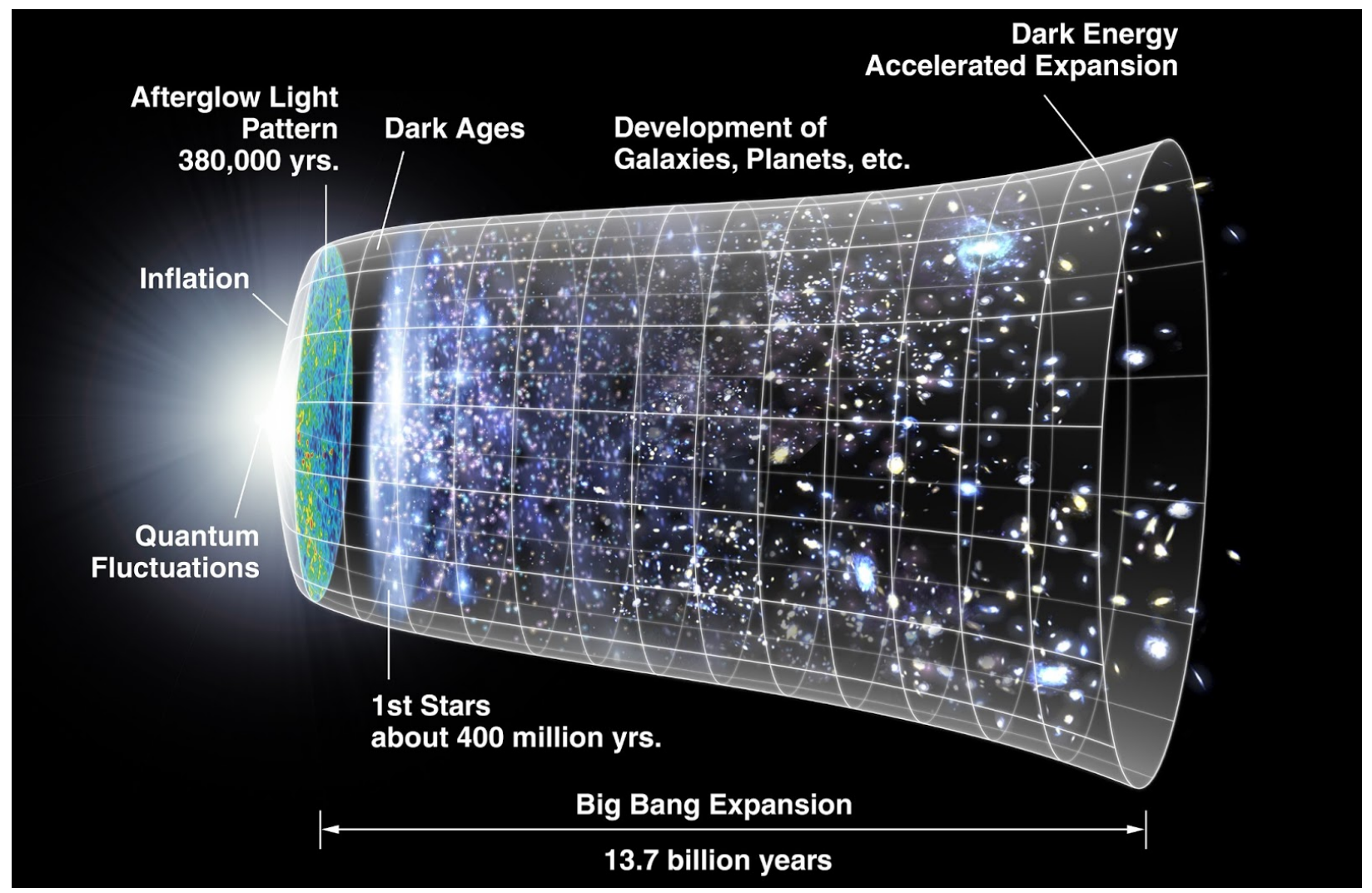

Figure 6: This timeline of cosmic evolution shows the flash of the big bang (left), latetime accelerated expansion, and the growth of structure in the intervening 13 billion years. The multi-colored map of the afterglow pattern on the left is the cosmic microwave background. The boundary of the observable universe is shown by the gridded cone. The changing shape represents changes in the expansion rate: note the periods of accelerated expansion: at very early times (left; inflation) and at late times (right; dark energy) [Figure credit: NASA/STSci.]

[File: cosmic_history.jpg]

[URL: http://2.bp.blogspot.com/zhxjPoVdbRg/UUzRKGvrtFI/AAAAAAAAAwo/Lxs0w0lzDEU/s1600/CMB Timeline300 no WMAP.jpg] 
It is important to add that the traditional correspondence between an open, closed or flat cosmos and the mass content of the universe is broken if dark energy exists. For example, with dark energy, it is possible to have a closed universe with an accelerating expansion. This is a very different understanding of the cosmos than one usually encounters in science popularizations.

\section{Alternatives to dark energy}

While dark energy is the simplest explanation for the accelerating expansion of the universe, there are alternative proposals. One idea that is a popular contender is the idea of quintessence. In contrast to dark energy, which is a constant energy density, quintessence is a dynamic energy field. Stated more simply, quintessence has many of the same properties as dark energy, but it can vary over cosmic time. In principle, it could remain similar, disappear entirely, or increase exponentially in the future. Each of these possibilities leads to very different predictions about the future of the cosmos.

While quintessence has conceptual commonalities with dark energy, there are alternative proposals to having a new form of energy. The simplest of these ideas is simply that general relativity is not an accurate theory at large distances. General relativity works extremely well and has passed a large number of experimental tests within the confines of our solar system. While modified gravity is generally less favored, it is still broadly acknowledged that we must include it as part of our experimental and theoretical programs until we are certain it is unnecessary. 
Other ideas are based on violations of the cosmological principle, or at least modifications of it. The cosmological principle states that, on sufficiently large distance scales, the universe is both homogeneous and isotropic; essentially, we do not inhabit a special region of the universe. Within our observations of the visible cosmos, this principle seems to be valid. While maps of the matter distribution of the universe do show clumpy structures, on size scales larger than about 250 million light-years, the distribution seems to be statistically isotropic, a point illustrated in figure 7 . Further, the microwave background radiation in the universe seems to be isotropic to about one part in $10^{5}$. This evidence supports the cosmological principle, although we continuously revisit and retest this assumption, as it is central to our theoretical thinking. It remains possible that density variations (i.e. large-scale structures) on length scales larger than the visible universe could explain the observed accelerating expansion of our universe, although this explanation is not a popular one.

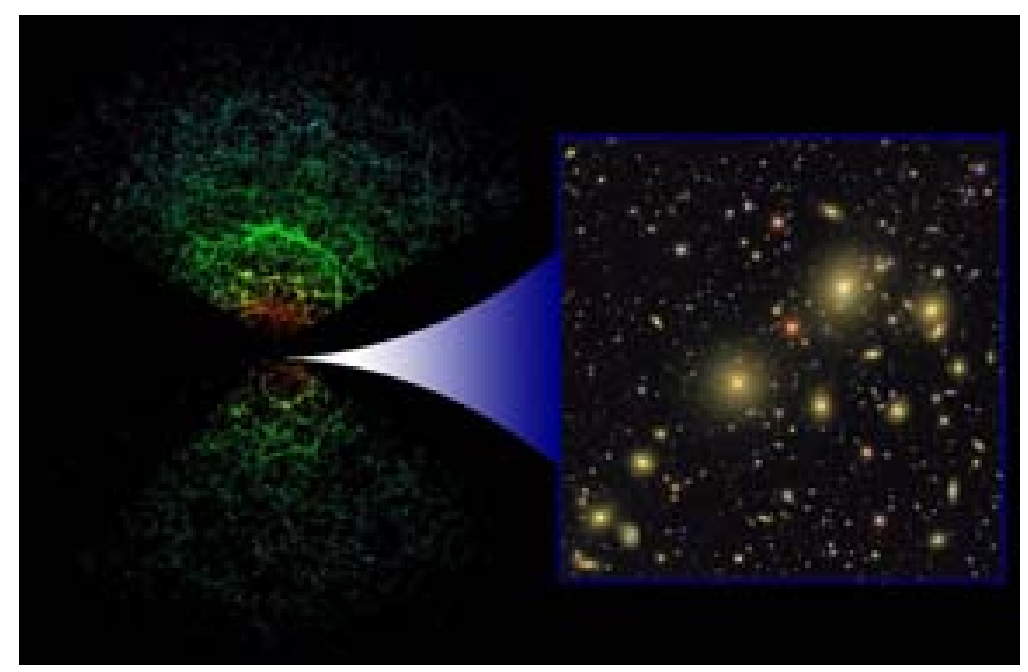

Figure 7: The survey area of the Sloan Digital Sky Survey displays the large-scale structure of the galaxy distribution, including individual galaxies (the smallest points), clusters of galaxies and the filaments that permeate between them. Earth is the 
observation point at the center, and distances (and time into the past) increase from the center. The inset shows an example small image of galaxies taken by the SDSS telescope. On the largest distance scales, the universe is statistically isotropic. [Figure credit: SDSS collaboration.]

[URL: http://www.sdss.org/news/releases/20031028.powerspectrum.html]

[File: SDSS_galaxy_locations.jpg]

\section{Experimental approaches to study dark energy}

In order to distinguish between the various models proposed to explain why the expansion of the universe is accelerating, it is crucial to make precise studies of many phenomena. To appreciate the diversity of the experimental program, it is necessary to describe the various measurements that can shed light on the situation.

1. Supernovae surveys: This approach is identical to those initially used to observe the accelerating expansion of the universe.

2. Surveys of galaxy populations: This method compares measurements of large numbers of galaxies (thousands to hundreds of millions) with predictions of the density of galaxies per unit of volume for various cosmological models. When the universe was smaller and hotter, sound waves were transmitted across the cosmos, with higher- and lower-density portions of the universe, just like how familiar sound is transmitted in air. However at about 380,000 years after the Big Bang, the universe cooled enough to decouple the matter and the sound waves, and the pattern of rarefication and concentration "froze," with a characteristic wavelength. Gravitational attraction amplified these variations in density in the 
early universe, resulting in variations in the location of galaxy formation. In essence, galaxies preferentially formed in regions of high density and were less likely to be formed in regions of low density, with separations that are easy to calculate. The expansion of the universe has caused these patterns of concentrations of galaxies to similarly expand. By studying the actual size of these patterns as a function of distance from Earth (and, consequently, time) tells us a lot of the history of the universe and how its size has varied. This technique is illustrated in figure 8.

3. Large-scale structure: among the structures in the universe are collections or clusters of galaxies, which are formed over billions of years via gravitational attraction. Dark energy can act against gravitational attraction, influencing the size and concentration of these clusters. When we measure the numbers of clusters at different times in the universe's history, this gives us a window into how strong dark energy was at those times.

4. Gravitational lensing: According to general relativity, matter can distort space. One of the consequences of that fact is that if you have two concentrations of mass, one distant and one closer, and the distant one emits light in your direction, the closer mass can distort the image of the more distant mass. This phenomenon is called gravitational lensing. While dark energy does not directly distort the passage of light, dark energy does affect the formation of large clusters of galaxies. By studying gravitational lensing as a function of the distance of the lensing masses, one can determine their distribution. Because of 
the link between dark energy and how mass accumulates over time, we can use these observations to constrain our models.

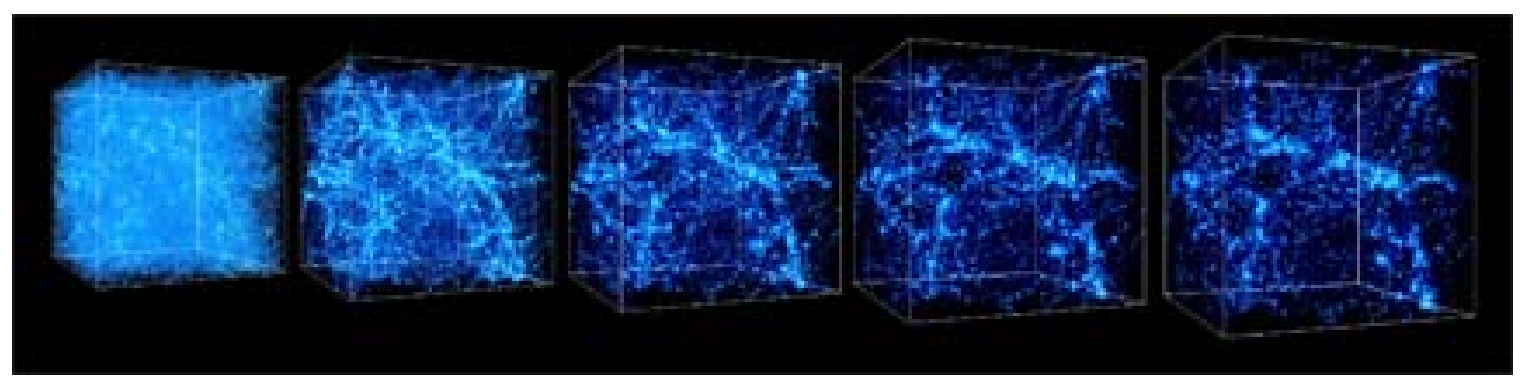

Figure 8: A simulation of the growth of large-structure of matter in the universe. At early times (far left), matter was distributed more evenly throughout the universe than today (right). As time grew on gravity's influence was revealed, as galaxies, filaments and clusters of galaxies formed. This is a computer simulation of dark matter evolving over cosmic time under the influence of gravity, and dark energy must be taken into consideration in the calculations. [Illustration credit: Center for Cosmological Physics/U Chicago]

[URL: http://cosmicweb.uchicago.edu/images/anim.jpg]

[File: universe_structure.jpg]

\section{Current and Future Experiments}

The techniques we just described are employed by several experiments that have either begun operations or will do so in the near future. We gather data on celestial objects, like galaxies and supernovae, by scanning the sky with large telescopes for many nights across several years. The Sloan Digital Sky Survey (SDSS) observed a quarter of the sky and characterized almost a million galaxies and hundreds 
of supernovae. The Dark Energy Survey (DES)[Reference:DES], which is about a year into its five-year mission, will increase those numbers by two orders of magnitude, reaching yet further into the past. The goal of DES is to detect the potentially varying nature of dark energy: i.e., if dark energy is quintessence. For regular updates on DES and easy-to-read descriptions of how we search for dark energy, check out DarkEnergyDetectives.org. Figure 9 shows this instrument. The Large Synoptic Survey Telescope (LSST) [Reference:LSST] will take this yet another step further, observing more objects and even deeper into the past 


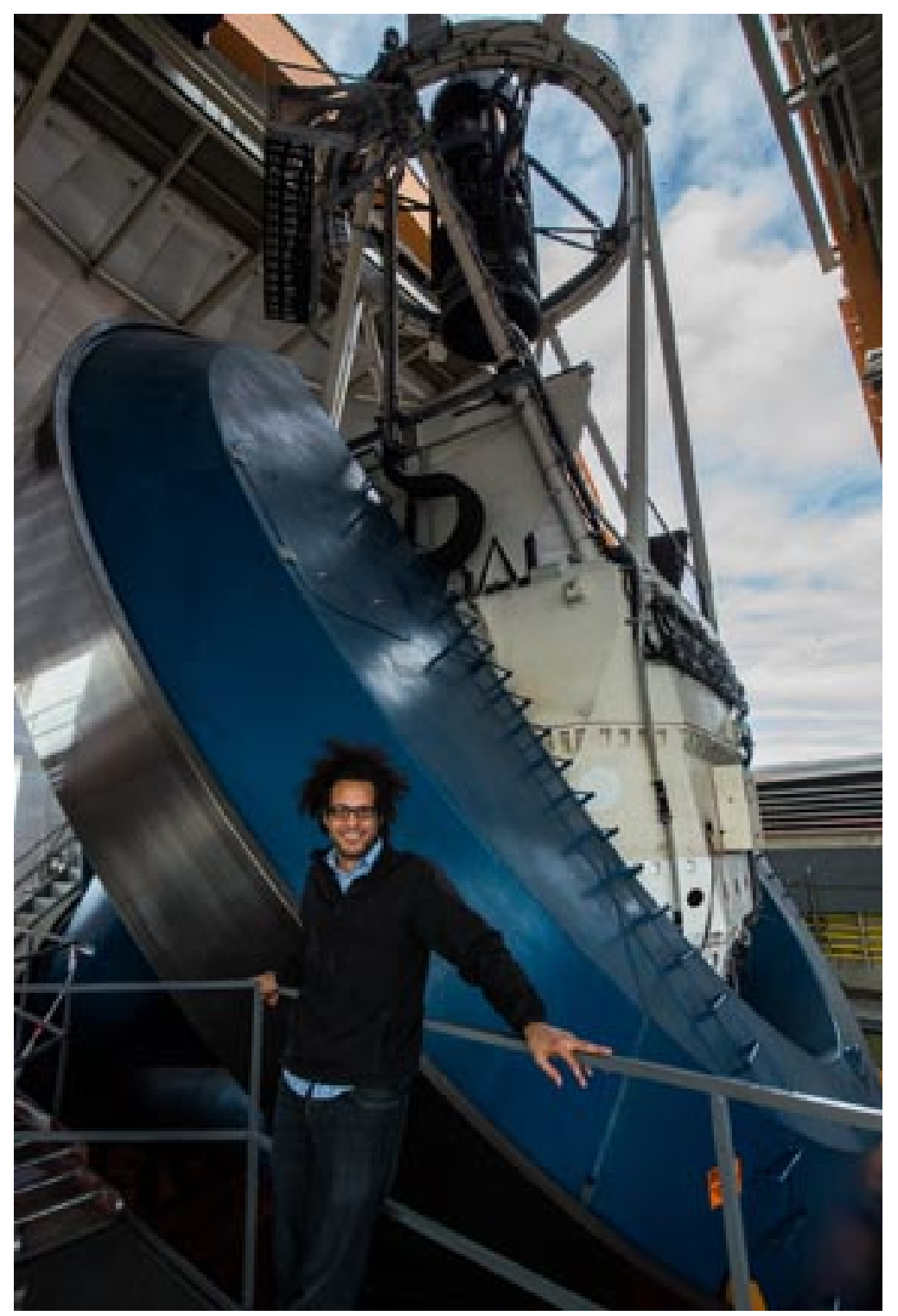

Figure 9: One of the authors (Nord) in front of the Dark Energy Survey telescope. [File: brian_telescope_medium.jpg]

These imaging surveys collect precise two-dimensional information on the plane of the sky about these objects and relatively poor information about their positions in the third dimension along the line of sight, the dimension that extends away from Earth and thus into the past. Spectroscopic surveys provide the highest-possible 3-dimensional 
measurement of positions. The Baryon Oscillation Spectroscopic Survey (BOSS, part of the SDSS campaign) [Reference: BOSS] was among the first of the major 3d surveys. The Dark Energy Spectroscopic Instrument (DESI) [Reference:DESI] will likely follow in the coming years and look even deeper into the past. Next-generation spectroscopic experiments will permit unprecedentedly precise measurements of how the universe expands, hopefully leading to the best chance we have of finding out if it changes in time.

\section{The next few years ... and eons}

Over the next few years, it is expected that experiments will be able to shed some light on dark energy, which appears to be the single largest ingredient in our universe. Is the cause of the accelerating expansion of the universe the constant energy density we call dark energy? Or is it quintessence, which varies in time? Or is it something else? If it is quintessence that increases over time, will the acceleration expand exponentially, with galaxies, stars, planets and even atoms ripped apart? Figure 10 shows some of these possible futures. 


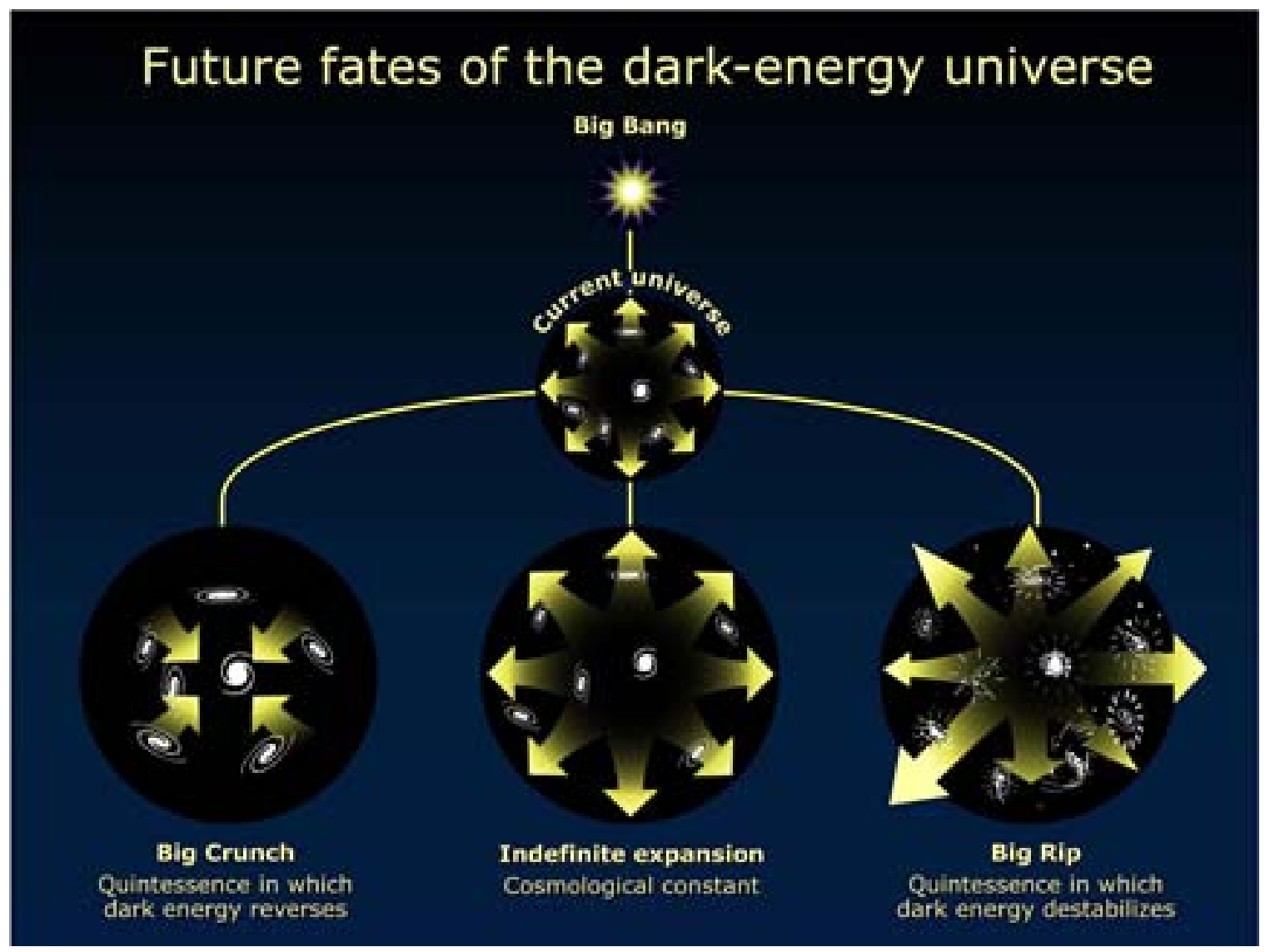

Figure 10: Some of the potential fates of our universe, from big crunch to big rip, caused by different kinds of dark energy. [Image credit: NASA/ESA and A. Riess $(\underline{\mathrm{STSCl}})]$

[URL: http://www.spacetelescope.org/static/archives/images/screen/opo0412c.jpg] [File: universe_fates.jpg]

Even if the simplest model of dark energy is true, the future of the universe is bleak. In many billion years, our galaxy will begin to merge with others in our local group of galaxies, first the Milky Way and Andromeda, perhaps then followed by the others. However, other galaxies at greater distances will be carried away by the expansion of space until they move outside our observable universe. In tens of billions 
of years, our descendants will live within a single galaxy formed by the merging of our local group, surrounded by darkness. The future promises to be a lonely, seemingly empty universe and we live in a privileged time to study dark energy, as a civilization that evolved billions of years from now would not observe distant galaxies and would imagine a much simpler cosmos. Research over the next several decades will confirm or falsify this prediction of the fate of the universe.

Carl Sagan is oft-quoted as saying "We are all star stuff." The elements of which we are made were forged in the core of aging stars and in the raging fires of supernovae. Even in more recent times, the dramatic death of stars has had a profound effect on humanity throughout our history by occasionally punctuating our night sky in ways that have been interpreted as messages from the heavens - harbinger of events yet to come. It is perhaps fitting that even in our more enlightened era, we, the children of supernovae, better understand those distant flashes of light and yet we still use them to predict the grandest future of them all - the evolution and the very fate of the universe.

\section{References}

[Reference:CMB] The Astrophysical Journal Supplement, Volume 208, Issue 2, article id. 19, 25 pp. (2013). (Equivalent URL is:

http://adsabs.harvard.edu/abs/2013ApJS..208...19H); http://map.gsfc.nasa.gov/]

[Reference:COBE] The Astrophysical Journal, Part 1 (ISSN 0004-637X), vol. 420, no. 2, p. 439-444 (Equivalent URL is http://adsabs.harvard.edu/abs/1994ApJ...420..439M); http://lambda.gsfc.nasa.gov/product/cobe/ 
[Referenc:Flat] http://map.gsfc.nasa.gov/universe/uni_shape.html

[Reference:Planck]arXiv:1303.5076(URL equivalent is

http://adsabs.harvard.edu/abs/2013arXiv1303.5076P);

http://www.rssd.esa.int/index.php?project=planck

[Reference:CFA]The Astrophysical Journal Supplement, Volume 208, Issue 2, article id. 19, 25 pp. (2013)(Equivalent URL is http://adsabs.harvard.edu/cgibin/bib_query?1978ApJ...221_...1D);

https://www.cfa.harvard.edu/ dfabricant/huchra/zcat/

[Reference:SDSS] THE ASTROPHYSICAL JOURNAL, 507:46Ė63, 1998 November 1(Equivalent URL is: http://iopscience.iop.org/0004-637X/507/1/46/pdf/0004637X_507_1_46.pdf); http://www.sdss.org/ [Reference:Dark Matter]Don Lincoln, Dark Matter, Physics Teacher, 51, 134 (2013). [Reference:SCP] The Astrophysical Journal, Volume 517, Issue 2, pp. 565586(Equivalent URL is http://adsabs.harvard.edu/abs/1999ApJ...517..565P); http://supernova.lbl.gov/

[Reference:High-Z] THE ASTROPHYSICAL JOURNAL, 507:46Ė63, 1998 November 1(Equivalent URL is http://iopscience.iop.org/0004-637X/507/1/46/pdf/0004637X_507_1_46.pdf;)

The Astronomical Journal, Volume 116, Issue 3, pp. 1009-1038 (URL equivalent is http://adsabs.harvard.edu/abs/1998AJ...116.1009R);

http://www.cfa.harvard.edu/supernova/HighZ.html

[Reference:DES] http://www.darkenergysurvey.org 
[Reference:LSST]arXiv:0912.0201 [Equivalent URL is

http://adsabs.harvard.edu/abs/2009arXiv0912.0201L ];

www.lsst.org

[Reference: BOSS] The Astronomical Journal, Volume 145, Issue 1, article id. 10, 41 pp. (2013) (URL equivalent is http://adsabs.harvard.edu/abs/2013AJ....145...10D); http://www.sdss3.org/index.php [Reference:DESI] http://arxiv.org/abs/1308.0847;

http://desi.lbl.gov/

Further Investigation

1. Iain Nicolson, Dark Side of the Universe: Dark Matter, Dark Energy, and the Fate of the Cosmos, Johns Hopkins University Press (2007).

2. Timothy Clifton and Pedro G. Ferreira, Does Dark Energy Really Exist, Scientific American, (April 2009).

3. http://www.youtube.com/watch?v=THT BAdKe6g

4. http://www.DarkEnergyDetectives.org

Possible cover art:

http://www-visualmedia.fnal.gov/VMS Site/gallery/stillphotos/2012/0300/12-032629D.jpg

HR version: http://www-visualmedia.fnal.gov/VMS Site/gallery/stillphotos/2012/0300/120326-29D.hr.jpg

Photo credit: Fermilab/Reidar Hahn 